\title{
BMJ Open Use of general practice before and after mild traumatic brain injury: a nationwide population-based cohort study in Denmark
}

\author{
Stine Fjendbo Galili, ${ }^{1}$ Bodil Hammer Bech, ${ }^{2}$ Claus Vestergaard, ${ }^{1}$ \\ Morten Fenger-Gron, ${ }^{1}$ Jakob Christensen, ${ }^{3}$ Mogens Vestergaard, ${ }^{1}$ \\ Jette Ahrensberg ${ }^{4}$
}

To cite: Galili SF, Bech BH, Vestergaard C, et al. Use of general practice before and after mild traumatic brain injury: a nationwide population-based cohort study in Denmark. BMJ Open 2017;7:e017735. doi:10.1136/ bmjopen-2017-017735

- Prepublication history for this paper is available online. To view these files, please visit the journal online (http://dx.doi org/10.1136/bmjopen-2017017735).

Received 15 May 2017 Revised 2 September 2017 Accepted 3 October 2017

\section{CrossMark}

${ }^{1}$ Department of Public Health, Research Unit for General Practice, Aarhus University, Aarhus, Denmark

${ }^{2}$ Department of Public Health, Research Unit for General Practice and Section for Epidemiology, Aarhus University, Aarhus, Denmark

${ }^{3}$ Department of Neurology, Aarhus University Hospital, Aarhus, Denmark

${ }^{4}$ Department of Clinical Medicine, Research Center for Emergency Medicine, Aarhus University Hospital, Aarhus, Denmark

Correspondence to Dr Stine Fjendbo Galili; s.galili@ph.au.dk

\section{ABSTRACT}

Objectives Traumatic brain injury (TBI) is commonly seen in the emergency department (ED). Approximately $85 \%-90 \%$ of TBls are mild (mTBI). Some cause symptoms such as headache, dizziness, anxiety, blurred vision, insomnia and concentration difficulties, collectively known as postconcussion syndrome (PCS). Some studies suggest that recovery from $m$ TBI is complete. Others find that symptoms persist for months, even years. The aim of this study was to describe the use of general practice, before and after mTBI, as a proxy for symptoms in a large cohort. Design Nationwide population-based matched cohort study.

Setting Danish EDs and general practice.

Participants All patients (aged $\geq 18$ years), first-time diagnosed with $\mathrm{mTBl}$ in a Danish ED between 1 January 1998 and 31 December 2010 ( $n=93517)$. Ten reference persons per patient with $\mathrm{mTBI}$ were randomly matched on gender, age and general practice $(\mathrm{n}=935170)$.

Primary outcome Overall use of general practice; consultations relating to mental and physical health. Results We found higher use of general practice during the first year after $\mathrm{mTBl}$ for all ages, both genders and all types of contacts. Age 18-40 years: women, incidence rate ratio (IRR) 1.59 (95\% Cl 1.57 to 1.61); men, IRR 1.82 (95\% $\mathrm{Cl} 1.80$ to 1.85 ). Age $41-65$ years: women, IRR 1.75 (95\% $\mathrm{Cl} 1.72$ to 1.78 ); men, IRR 1.85

(95\% Cl 1.82 to 1.89 ). Age $66+$ years: women, IRR 1.55 (95\% Cl 1.52 to 1.58 ); men, IRR 1.55 (95\% Cl 1.51 to 1.59). After the first year, the use decreased to the level before $\mathrm{mTBI}$. Individuals with $\mathrm{mTBI}$ and higher use of general practice before mTBI had lower socioeconomic status and more comorbidities $(\mathrm{P}<0.001)$.

Conclusions The use of general practice was higher in the first year after mTBI, specifically in the first 3 months. Patients with mTBI had different healthcare-seeking behaviour several years before diagnosis than their matched reference persons. Pretraumatic morbidity should be considered in the evaluation of PCS.

\section{INTRODUCTION}

Traumatic brain injury (TBI) is common at the emergency department (ED). TBIs
Strengths and limitations of this study

- This nationwide population-based matched cohort study was based on data from several Danish national registers.

- A major strength was the low risk of selection bias and information bias due to complete follow-up and high data quality.

- We adjusted for several potential confounders through linkage of national registers with updated information.

- No data on trauma mechanisms were available.

- The study was based on hospital admission and/or contact to $\mathrm{EDs}$, which may reduce the generalisability of the results.

account for over 1 million annual ED visits in the USA, ${ }^{1} 150000$ visits in the $\mathrm{UK}^{2}$ and 20000 visits in Denmark. ${ }^{3}$

Some TBIs may cause severe intracerebral lesions, which may call for neurosurgical intervention. A total of $85 \%-90 \%$ of TBIs are classified as mild (mTBI) ${ }^{3-5}$ which is defined as a condition where loss or suspected loss of consciousness, is short and a score of 14-15 on the Glasgow Coma Score (GCS). ${ }^{4} 67$ mTBI may be associated with symptoms such as headache, dizziness, irritability, anxiety, blurred vision, insomnia, fatigue and concentration/memory difficulties. ${ }^{8}$ It has been claimed that symptoms following mTBI are transient. ${ }^{9}$ Several studies suggest that the impact is small and recovery from mTBI is generally complete. ${ }^{10-12}$ However, symptoms following the mTBI may persist for weeks or months ${ }^{813-15}$ or even years. ${ }^{2} 1617$ These are collectively known as postconcussion syndrome (PCS).$^{18}$

PCS has been described in a number of publications, but relatively little is known about the prevalence and incidence. However, 
it has been estimated that $25 \%$ of all patients with mTBI suffer from postconcussive symptoms or other cognitive sequelae for more than a year after the mTBI. ${ }^{19}$

Several risk factors for PCS have been identified, including a history of multiple mTBIs, higher age, female sex, pre-existing pain and psychiatric and psychological comorbidities. ${ }^{1720}$

Previous studies have indicated that mTBI is associated with decreased quality of life, ${ }^{15}$ long-term sick leave ${ }^{21}$ and unemployment. ${ }^{22}$ However, as inclusion criteria differ and many studies have relatively small sample sizes it is difficult to compare and conclude across studies.

Healthcare-seeking behaviour patterns after mTBI may provide new knowledge on the PCS period, and increased use of general practice can thus be seen as a proxy variable for symptom presentation.

The structure of the Danish healthcare system requires the patients to initially contact the general practitioner (GP) who may refer the patient to a specialist (here: a privately practicing neurologist). This type of service is also covered by the taxes levied by the state and is thus free of charge for the patients. The aim of this large study was to describe the use of primary healthcare before and after mTBI as a proxy for symptoms and concerns among patients suffering from mTBI.

\section{METHODS}

This nationwide population-based matched cohort study was based on data from several Danish national registers: the Danish Civil Registration System (CRS) ${ }^{23}$ which holds updated information on all Danish citizens, the Danish National Hospital Register (NHR), which contains nationwide data on all somatic hospital admissions from 1977 onwards, (data on outpatients activities and emergency visits have been included since 1995), ${ }^{24}$ the Danish National Health Insurance Service Register (NHSR), which holds information on all contacts to general practice and all services provided, ${ }^{25}$ the Danish Register of Medicinal Product Statistics (RMPS) ${ }^{26}$ and Statistics Denmark, which holds information on income, marital status and education. ${ }^{27}$

The Danish civil registration number, which is a unique 10-digit personal identification number assigned to all Danish citizens at birth or immigration, was used to link information at the individual level across the registers. ${ }^{28}$

\section{Study population}

All adult patients (aged $\geq 18$ years) first-time diagnosed with mTBI in a Danish ED between 1 January 1998 and 31 December $2010(\mathrm{n}=93517)$ were identified in the NHR (ICD-8: 850.99; ICD-10: S06.0). ${ }^{28}$

The CRS was used to randomly sample 10 reference persons per patient with mTBI $(\mathrm{n}=935170)$. Reference persons were matched on gender and age, and also on general practice, allowed using an algorithm linking individual patients to their general practice. ${ }^{29}$ The reference persons had no former diagnosis of mTBI or other more severe TBI (ICD-10: 06.1-06.9) ${ }^{30}$ on the day of diagnosis with mTBI. A reference person could be sampled only once per case, but a reference person could act as reference for multiple cases and could be included as a patient with $\mathrm{mTBI}$ later in the study period.

Estimates were adjusted for the following comorbidities: hypertension, ischaemic heart disease, heart failure, stroke, epilepsy, alcohol problems, substance abuse and dementia. Medical comorbidities were derived from a multimorbidity index ${ }^{31}$ comprising 39 psychiatric and physical long-term conditions; this index has previously been used in a Danish setting. ${ }^{31}$ Additionally, comorbidities were based on information from the $\mathrm{NHR}^{24}$ and the RMPS. ${ }^{26}$ Information on socioeconomic characteristics was obtained from Statistics Denmark. ${ }^{27}$

Both patients with mTBI and reference persons were residents in Denmark and listed with a Danish general practice from 5 years before and up to 10 years after the mTBI. Both patients with mTBI and reference persons were censored by death $(\mathrm{n}=139850)$, disappearance $(\mathrm{n}=347)$ or emigration $(\mathrm{n}=11053)$.

\section{Outcome measurements}

The overall use of general practice 5 years before and up to 10 years after mTBI was applied as outcome measure. This included all types of daytime and out-of-hours contacts: face-to-face consultations, telephone consultations and email consultations. Furthermore two services relating to mental and physical health during the same period were included as outcome measure: talk therapy in general practice and contact to privately practicing neurologist.

\section{Statistical analysis}

For both persons with mTBI and reference persons, we estimated the monthly number of contacts with general practice (in the 12 months before and the 12 months after the mTBI diagnosis) and the mean number of contacts per year (in the 5 years before and up to 10 years after the mTBI) with corresponding $95 \%$ CIs. We calculated incidence rate ratios (IRRs) and adjusted the analyses for comorbidities and socioeconomic relations by using a negative binomial regression model including cluster robust variance estimation. Subgroup analyses were performed to stratify for age groups and gender. A $P$ value of $<0.05$ was defined as statistically significant. All data handling and statistical analysis were performed with Stata V.13.1 statistical software (StataCorp LP, Texas, USA).

\section{RESULTS}

\section{Characteristics of the study population}

Baseline characteristics of the 93517 included patients with mTBI and their 935170 matched reference persons are shown in table 1.

Persons with mTBI were more likely to live alone (unmarried, divorced or widowed), were less educated, 
Table 1 Characteristics of the study population

\begin{tabular}{|c|c|c|}
\hline & $\begin{array}{l}\text { Patients with } \\
\text { mTBI }\end{array}$ & References \\
\hline$n$ & 93517 & 935170 \\
\hline \multicolumn{3}{|l|}{ Sex (\%) } \\
\hline Female & 42789 (45.8) & 427890 (45.8) \\
\hline Male & $50728(54.2)$ & $507280(54.2)$ \\
\hline $\begin{array}{l}\text { Age, mean, years } \\
\text { (SD) }\end{array}$ & $45.9(21,2)$ & $46.0(21,2)$ \\
\hline \multicolumn{3}{|l|}{ Age group (\%) } \\
\hline $18-40$ years & 44709 (47.8) & 447080 (47.8) \\
\hline 41-65years & 29761 (31.8) & 297545 (31.8) \\
\hline$>66$ years & 19047 (20.4) & 190545 (20.4) \\
\hline \multicolumn{3}{|l|}{ Education (\%) } \\
\hline$\leq 10$ years & 39428 (42.3) & 339573 (36.3) \\
\hline$>10$ and $\leq 15$ years & 36201 (38.7) & $401613(42.9)$ \\
\hline >15years & $10450(11.2)$ & 124841 (13.3) \\
\hline Unknown & $7338(8,0)$ & $69143(7,4)$ \\
\hline \multicolumn{3}{|l|}{ Marital status (\%) } \\
\hline Married & 30223 (32.3) & $382481(40,9)$ \\
\hline Widowed & 10044 (10.7) & 89328 (9.6) \\
\hline Divorced & 11502 (12.3) & 72912 (7.8) \\
\hline Unmarried & $41748(44.6)$ & 390448 (41.8) \\
\hline \multicolumn{3}{|l|}{ Income (\%) } \\
\hline Low & 25152 (26.9) & $236282(25.3)$ \\
\hline Medium & $47256(50.5)$ & 427495 (45.7) \\
\hline High & 21109 (22.6) & 271393 (29.0) \\
\hline \multicolumn{3}{|l|}{ Comorbidities (\%) } \\
\hline Hypertension & 12796 (13.7) & 121206 (13.0) \\
\hline Heart failure & $910(1.0)$ & $7152(0.8)$ \\
\hline Stroke & 2691 (2.9) & $16.166(1.7)$ \\
\hline Epilepsy & $1283(1.4)$ & $4248(0.5)$ \\
\hline Alcohol abuse & $3360(3.6)$ & $4087(0.4)$ \\
\hline Drug abuse & $884(0.9)$ & $1837(0.2)$ \\
\hline Dementia & $1005(1.1)$ & $5558(0.6)$ \\
\hline
\end{tabular}

mTBI, mild traumatic brain injury.

had lower income and more often had comorbidities compared with the reference persons (table 1).

Women generally had higher use of general practice than men; women in the youngest age group had two times as many contacts as men (figure 1).

\section{Consultations in general practice before and after mTBI}

When analysing the number of contacts to general practice 5 years before the mTBI diagnosis and 10 years after, we found a higher number of contacts for both men and women with mTBI compared with the reference persons (figure 1, upper part).

Persons with mTBI had more contacts to general practice both before and after the mTBI. The IRR for contacts to general practice was generally higher from the date of mTBI and the following year (figure 1). In the youngest age group, the IRR for women was $1.59(95 \%$ CI 1.57 to 1.61$)$, whereas the number of contacts was almost two times as high for men with mTBI compared with reference persons, as the IRR was 1.82 (95\% CI 1.80 to 1.85 ) in the year following the mTBI. In the middle-aged group, the IRR for women was 1.75 (95\% CI 1.72 to 1.78 ), whereas the IRR for men was 1.85 (95\% CI 1.82 to 1.89 ). In the oldest age group, the IRRs were identical for both genders with an IRR 1.55 (95\% CI 1.52 to 1.58 ) for women and an IRR of 1.55 (95\% CI 1.51 to 1.59 ) for men. Five years after the mTBI diagnosis, the IRRs had fallen and remained at the same level as 5 years before mTBI diagnosis. The patients with mTBI had more contacts to general practice compared with the reference persons in all age groups; this was seen for both genders from 5 years before the mTBI.

The number of contacts peaked during the first 3 months after the mTBI, whereafter the number of contacts decreased (figure 2).

In the youngest age group, the adjusted number of contacts for women was almost three times higher in the mTBI group than in the reference group during the first month after mTBI diagnosis (IRR 2.88, $95 \%$ CI 2.74 to 3.02 ). Men in the mTBI group had more than four times as many contacts during the first month (IRR 4.34, 95\% CI 4.13 to 4.56). In the middleaged group, the adjusted number of contacts was more than three times higher in the mTBI group for women (IRR 3.48, 95\% CI 3.32 to 3.66 ) and four times higher for men (IRR 4.06, 95\% CI 3.87 to 4.27 ) during the first month after mTBI. In the oldest age group, the IRRs in the first month were lower and similar for men (IRR 2.42, 95\% CI 2.3 to 2.54 ) and women (IRR $2.46,95 \%$ CI 2.34 to 2.59 ). The increasing number of contacts to general practice for patients with mTBI, was observed during the 3 months after mTBI, after which the number of contacts fell to almost the level before the mTBI. The decrease in number of contacts after mTBI was seen in all age groups and for both genders.

\section{Talk therapy in general practice, before and after mTBI}

Persons in both the youngest and the middle-aged group with mTBI had significantly higher use of talk therapy in general practice before mTBI compared with the reference persons (figure 3). The data show that patients with mTBI had almost two times as many contacts involving talk therapy in general practice before the mTBI. In the reference group, $6.37 \%(95 \%$ CI $6.31 \%$ to $6.42 \%$ ) had a contact to general practice involving talk therapy. For the persons with mTBI, $11.47 \%$ (95\% CI $11.29 \%$ to $11.64 \%$ ) had this type of contact before the mTBI.

In the youngest age group, both men and women had about two times as many contacts involving 


\section{All contacts}
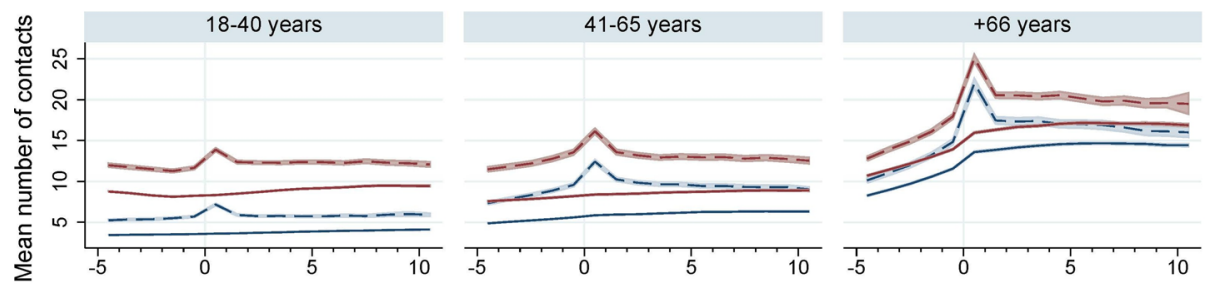

Years relative to $\mathrm{mTB}$

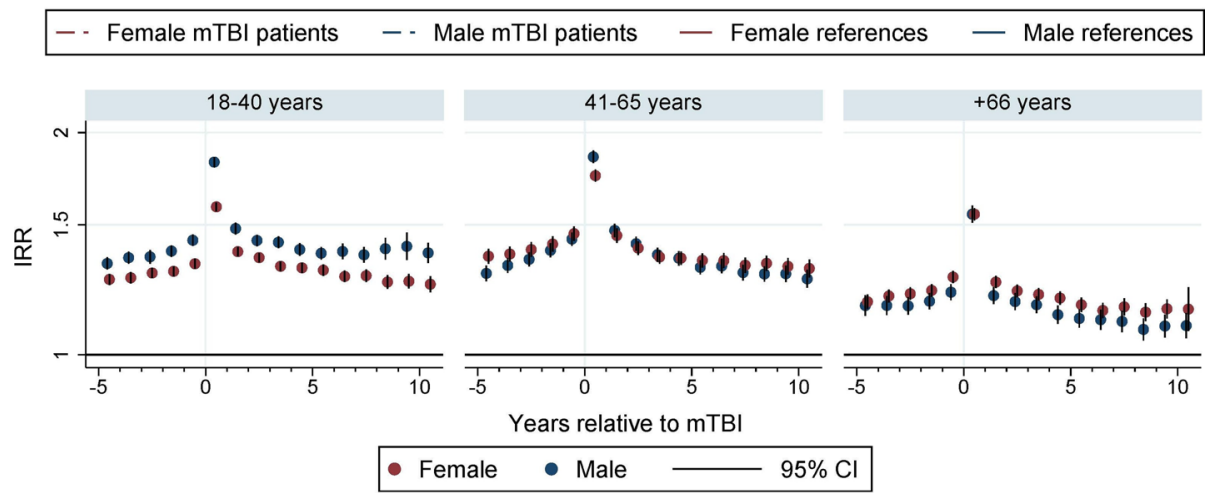

Figure 1 All contacts in general practice before and after mild traumatic brain injury (mTBI) diagnosis. Upper part: mean number of contacts and $95 \%$ Cls for all contacts in general practice for persons with $\mathrm{mTBI}$ and reference persons, 5 years before and 10 years after the mTBI diagnosis. Lower part: incidence rate ratios (IRRs) for all contacts with 95\% Cls adjusted for comorbidities and socioeconomic status.

talk therapy in general practice than the reference persons (women: IRR $1.96,95 \%$ CI 1.82 to 2.12 ; men: IRR $2.15,95 \%$ CI 1.96 to 2.35 ) in the year leading up to the mTBI and in the year following the mTBI. In the middle-aged group, a peak was seen in the first year after the mTBI; both women (IRR 2.12, 95\% CI
1.86 to 2.41 ) and men (IRR 2.56, 95\% CI 2.27 to 2.88 ) had more than two times as many contacts involving talk therapy in general practice compared with the reference persons. In the oldest age group, no increase in talk therapy in general practice was seen around the time of diagnosis (women: IRR 1.52, 95\% CI

\section{All contacts}
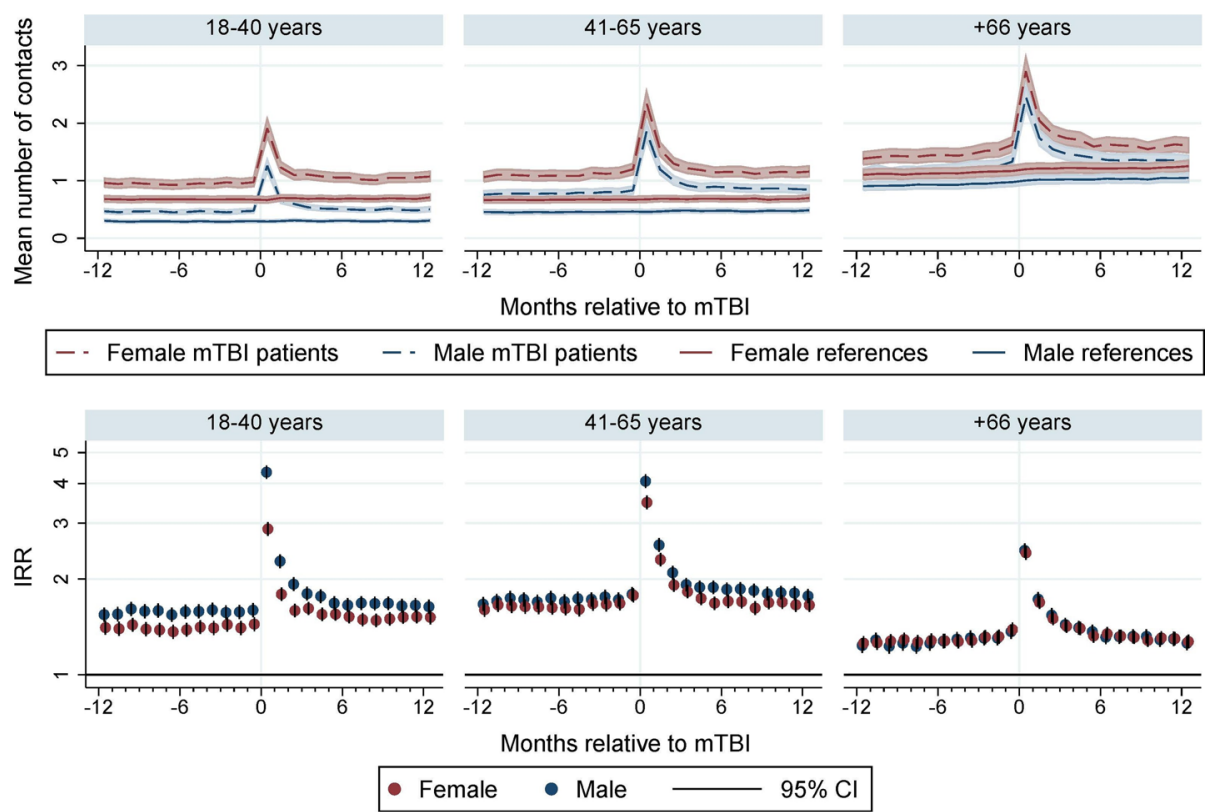

Figure 2 All contacts to general practice 12 months before and 12 months after mild traumatic brain injury (mTBI). Upper part: mean number and $95 \% \mathrm{Cls}$ for all contacts to general practice for persons with $\mathrm{mTBI}$ and reference persons, 12 months before and 12 months after mTBI diagnosis. Lower part: incidence rate ratios (IRRs) for all contacts with $95 \%$ Cls adjusted for comorbidities and socioeconomic status. 
Talk therapy in general practice
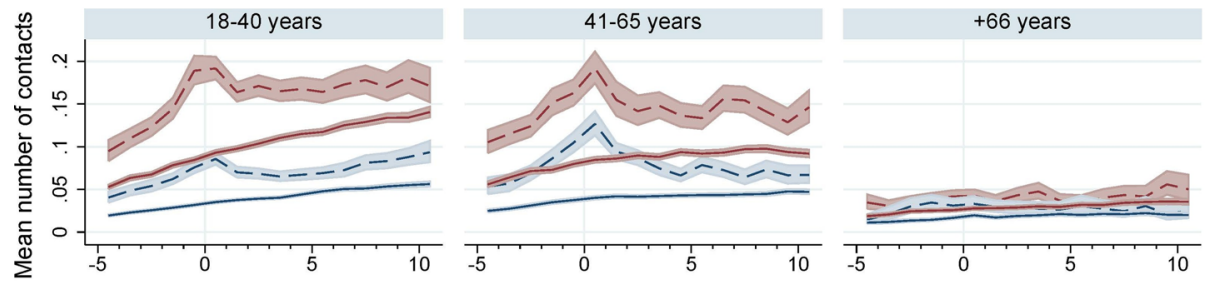

Years relative to $\mathrm{mTB}$

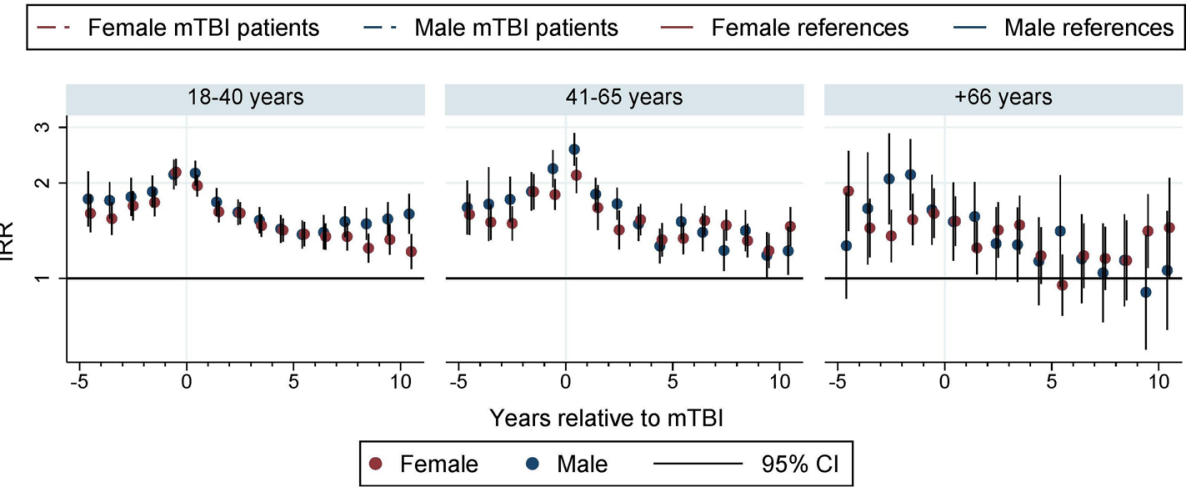

Figure 3 Talk therapy in general practice before and after mild traumatic brain injury (mTBI). Upper part: mean number of and 95\% Cls for contacts including talk therapy in general practice for persons with mTBI and reference persons 5 years before and 10 years after the diagnosis. Lower part: incidence rate ratios (IRRs) for contacts including talk therapy in general practice with 95\% Cls adjusted for comorbidities and socioeconomic status.

1.27 to 1.81 , men: IRR $1.51,95 \%$ CI 1.14 to 2.01$)$. However, persons with mTBI generally had higher use of talk therapy in general practice than the reference persons throughout the observation period. During the first year after the mTBI diagnosis, the IRR fell to a level below the IRR before the mTBI for both gender and all age groups.

\section{Consultations with a privately practicing neurologist before and after $\mathrm{mTBI}$}

Both men and women with mTBI had more consultations with a privately practicing neurologist compared with reference persons at the time of the mTBI diagnosis and during the year after (figure 4). Furthermore, we found that $4.89 \%$ (95\% CI $4.78 \%$ to $5.01 \%$ ) of patients with $\mathrm{mTBI}$ had contact to a privately practicing neurologist before the mTBI diagnosis, whereas $6.97 \%$ (95\% CI $6.85 \%$ to $7.08 \%$ ) had contact after the mTBI diagnosis. In the reference group, the corresponding numbers were $3.15 \%$ before (95\% CI $3.11 \%$ to $3.19 \%$ ) and $3.18 \%$ after (95\% CI $3.14 \%$ to $3.21 \%$ ) the date of the mTBI diagnosis.

In the youngest age group, women with mTBI had more than four times as many contacts, (IRR $4.55,95 \% \mathrm{CI}$ 4.11 to 5.03 ) during the first year after mTBI diagnosis, and men had more than five times as many contacts (IRR $5.54,95 \%$ CI 4.95 to 6.21 ) to a privately practicing neurologist than the reference persons. In the middle-aged group, the number of consultations had increased similarly for both women and men (women: IRR 4.69, 95\% CI 4.21 to 5.23, men: IRR $4.01,95 \%$ CI 3.58 to 4.49$)$. In the oldest age group, the relative increase in number of neurological consultations was smaller (women: IRR 2.40, 95\% CI 2.06 to 2.80; men: IRR $1.8195 \%$ CI 1.45 to 2.20 ).

\section{DISCUSSION}

\section{Main findings}

This large nationwide population-based study identified higher use of general practice for up to 3 months after an mTBI diagnosis compared with a reference group. Hereafter, the level decreased to approximately the level before the mTBI. The increase was observed for both genders, all age groups and all types of contacts to general practice. Persons with mTBI had more consultations in general practice involving talk therapy and more consultations with a privately practicing neurologist than their matched reference persons.

We also found that individuals with mTBI had higher use of general practice several years before the mTBI. This indicates that persons exposed to mTBI have a different healthcare-seeking behaviour and a higher comorbidity burden than their matched reference persons. These findings are supported by their higher use of talk therapy in general practice, more consultations with a privately practicing neurologist and the descriptive data on their socioeconomic characteristics.

\section{Strengths and limitations}

This population-based study achieved high statistical precision owing to the large study population. We established a cohort of all patients diagnosed with mTBI at any Danish hospital during a 12-year period and a random sample of 
Privately practicing neurologist
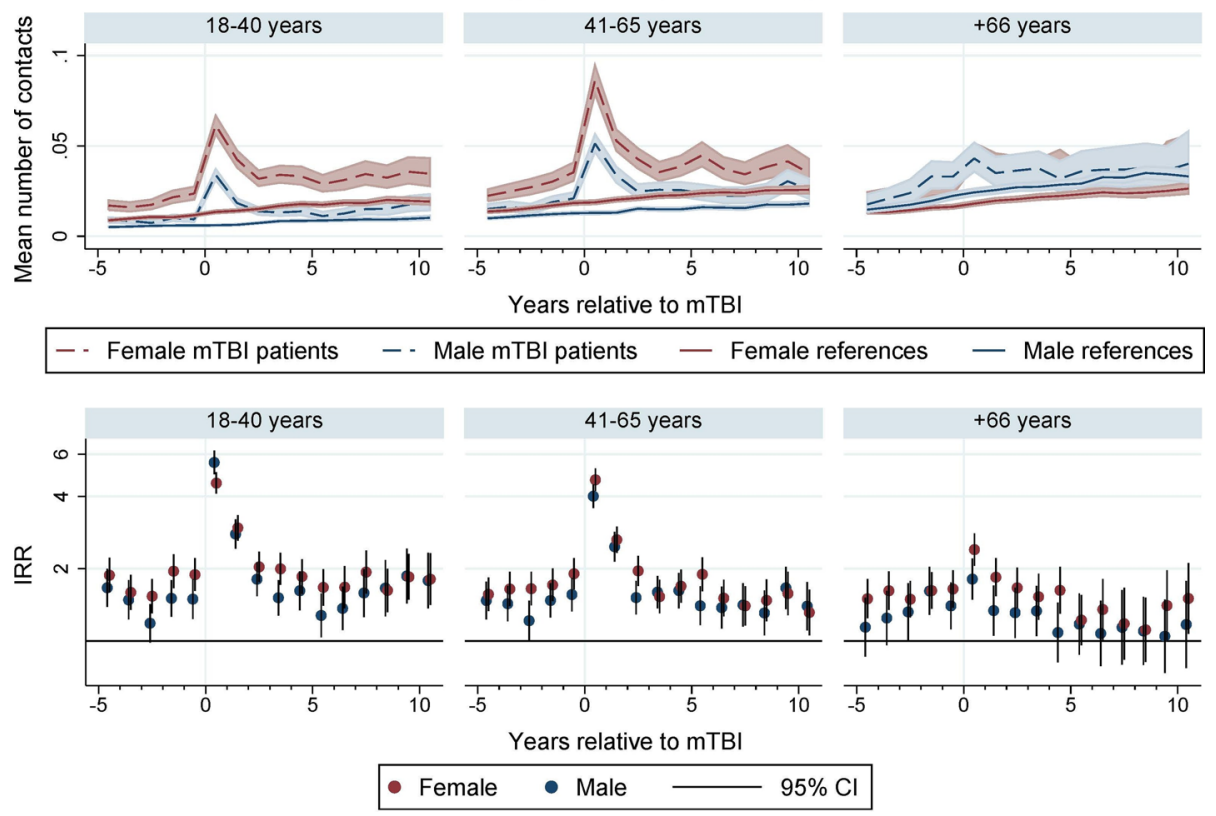

Figure 4 Consultations with a privately practicing neurologist before and after mild traumatic brain injury (mTBI). Upper part: mean number and $95 \%$ Cls for consultations with a privately practicing neurologist for persons with mTBI and reference persons 5 years before and 10 years after the mTBI diagnosis. Lower part: incidence rate ratios (IRRs) for consultations at a privately practicing neurologist with $95 \%$ Cls adjusted for comorbidities and socioeconomic status.

reference persons matched on gender, age and listed with the same general practice. We followed the cohort for up to 10 years with complete follow-up. Selection bias may, therefore, not explain our results. The data on healthcare use were not collected for the purpose of this study and were independent of the memory of the GP and the study participants. Consequently, the study is not likely to have been affected by recall bias. The registration of contacts to the general practice in the NHSR ${ }^{25}$ is considered to have high validity as the GP are reimbursed on the basis of these registrations which are checked by the authorities. On this background, we believe that information bias is an unlikely explanation for our findings. All diagnostic tests and consultations measured in the study were provided or requested by GPs and were available to the patients free of charge.

Patients with mTBI and reference persons were matched on demographic factors, age and gender, which further minimised potential bias. As the diagnosis of mTBI in all Danish EDs is based on the same criteria (ICD-8 or ICD-10), ${ }^{28}$ the case population can be perceived as homogeneous.

In the analyses, adjustments were made for relevant comorbidities and socioeconomic status on the basis of information from the $\mathrm{NHR}^{24}$ and Statistics Denmark ${ }^{27}$ to further reduce the risk of confounding. However, residual confounding from unmeasured factors cannot be completely excluded.

One of the limitations of this study is that no data on trauma mechanisms were available. The trauma itself could cause confounding by indication as the symptoms of PCS includes symptoms similar to depression or post-traumatic stress syndrome. ${ }^{32}$

The nationwide approach allows us to generalise the results to other countries with a healthcare system comparable to the Danish healthcare system, for example, the Nordic countries; however, this study was based on hospital admission and/or contact to EDs. This may reduce the generalisability of the results as patients with less severe injuries may not present for assessment and medical treatment. ${ }^{33}$ Hence, it is estimated that around half of patients sustaining mTBI have no loss of consciousness and no need for hospitalisation or contact to ED. ${ }^{34}$

We observed higher mortality rates in the mTBI group compared with reference persons. If the weakest and most severely ill of patients with mTBI dies (from complications), this could have caused the mTBI groups to appear healthier than it actually was. This would have skewed the results towards the null. This is unlikely to be problematic in the youngest age groups as the overall mortality is very low in these groups $(0.8 \%$ and $5.3 \%)$, but it could be a factor of importance in the oldest age group (40.2\%).

\section{Comparison with other studies}

Our characteristics of persons with mTBI correlate with those in a previous study, ${ }^{35}$ and our findings are in line with studies reporting that mTBI is a condition that disproportionately affects younger men ${ }^{36}$ and with meta-analyses showing that symptoms persist in many patients until approximately 3 months post injury. ${ }^{911}$ Likewise, the characteristics of both patients with mTBI and their matched reference persons confirm the findings of 
previous studies reporting that women in general (especially in the youngest group), have a higher use of general practice than men. ${ }^{37} 38$

The identified healthcare-seeking patterns suggest that the general course of symptoms following mTBI is no longer than 3 months, even though other studies describe symptoms beyond this period..$^{16} 20$ In this study, the use of general practice was seen as a proxy for symptom presentation, and also as an indicator of a patient's degree of discomfort or concern. It would be expected that persons with mTBI who have been suffering from PCS for more than 3 months would have shown in the number of contacts to the general practice and in the adjusted IRRs. Such increased utilisation of general practice was not observed in this study. However, the study revealed an increase in consultations with privately practicing neurologists for persons with mTBI. This indicates a need for more extensive medical investigation and possibly treatment.

Furthermore for contacts including talk therapy in general practice, we found that persons with mTBI generally have higher use, but this was not relating to mTBI in the youngest and oldest age group. During the first year after an mTBI the use of talk therapy in general practice decreased. This could suggest that the treatment may cease while the physical symptoms persist, and thus the mental health may be ignored in these months or even years.

Recent studies show a prolonged period of recovery for patients with mTBI. ${ }^{216} 39$ Patients in these studies were all examined in an ED setting and met the criteria for mTBI based on a CT scan or had a GCS of 13-15. Patients were assessed using different, objective and subjective scoring systems over multiple time points. These studies found that full recovery might not be achieved and they conclude that recovery from mTBI should be tracked past the conventional 12 months. ${ }^{2}{ }^{16}$ All studies found that many of the patients with persistent symptoms had a medical history or psychiatric problems, previous neurological condition, previous TBI or drug abuse. This could indicate that patients with $\mathrm{mTBI}$ and comorbidities have a higher risk of developing PCS. ${ }^{16}$ This correlates well with our descriptive data of patients with mTBI and our finding that persons with mTBI had higher use of general practice before the mTBI.

Different inclusion criteria for cases or smaller sample sizes may add to the discrepancy in the persistence of symptoms. The use of individual symptoms and several outcome measures over multiple time points provide a more global patient status and ensure capture of the more subtle sequelae of mTBI, but results are difficult to compare without a control group. ${ }^{21639}$ When subjective values are used, it should also be taken into consideration that PCS symptoms are difficult to distinguish from, for example, post-traumatic stress syndrome or depression /anxiety.
In our study, the inclusion criterion is the diagnosis commotio cerebri (ICD-8 and ICD-10), ${ }^{28}$ which is more detailed than using the GCS.

Postmortem studies of patients with mTBI have demonstrated pathological changes that are undetectable by conventional neuroimaging studies. ${ }^{40}$ Furthermore the pathophysiology of PCS is widely discussed and seems to be a combination of physical and psychogenic factors, possibly in the presence of the predisposing factors described in this study. Others using specialised imaging modalities (such as single-photon emission CT, MRI or electrophysiology) have shown the presence of persistent organic brain injury, in some cases up to 1 year after the mTBI. ${ }^{19} 204041$

The development of PCS may be due to a combination of several factors such as trauma mechanisms, pre-existing vulnerabilities and brain dysfunction.

Even though this study, which is based on healthcare utilisation, suggests that patients with mTBI do not have increased use of general practice for more than 3 months, we must still consider the possibility that these patients continue to have symptoms even though their healthcare-seeking is not increased. It has been suggested that one-quarter of patients may suffer from symptoms or have cognitive deficits for more than a year. ${ }^{19}$ However, patients may not seek help from the GP for these symptoms, as they believe that they cannot be relieved of the symptoms with the help from the GP.

In 2010, the economic burden of TBI in the USA, direct medical and indirect costs such as loss of productivity, was estimated to be US $\$ 76.5$ billion. Ninety per cent of this estimate is related to severe TBI hospitalisations. ${ }^{42}$ However, mTBI can also be associated with significant ongoing costs in terms of disability, lost work or neuropsychiatric complications, which was not analysed in this study. However, our results suggest that mTBI may be associated with increased utilisation of medical care for a minimum of 3 months after mTBI.

\section{Conclusions and implications}

This study shows increased use of general practice up to 3 months after an mTBI, this suggests that the symptoms may persist for at least this period. Our large dataset could not detect increased healthcare-seeking after this point indicating that most persons do not seem to need long-term help from the GP for mTBI induced symptoms. However, some patients may still have considerable problems without seeking help or advice in general practice.

Our study shows that patients seen in the EDs in Denmark for mTBI have a different healthcare-seeking behaviour for several years before the diagnosis of mTBI.

More profound identification of premorbid factors is called for to ensure optimal treatment and support for patients with mTBI. Attention should be paid to the mental well-being of these patients since we see a fall in consultations including talk therapy during the year following an mTBI. 
The aetiology of PCS seems to be multifactorial and appears to be caused by a combination of both the physical impact of mTBI and premorbid psychogenic factors. To ensure that the mental and physical state of these patients is not neglected, it may be valuable to offer these a follow-up consultation in general practice 3 or 6 months after the mTBI to investigate whether persons with mTBI suffer from subtle sequelae that is not presented to the GP.

This study calls for further research into the sequelae from mTBI, particularly pretrauma morbidity, injury-related and postinjury-related neuropathological hallmarks and psychological factors that may contribute to the development of PCS.

Contributors JA, BHB and MV initiated the study and obtained the funding. All authors participated in the design of the study. CHV performed the statistical analyses in a dialogue with BHB, MF-G and SFG. SFG wrote the manuscript. All authors were involved in the interpretation of data and the critical revision of the manuscript. SFG had the primary responsibility for the final content. All authors read and approved the final manuscript.

Funding This work was supported by the Program for Clinical Research Infrastructure (PROCRIN) established by the Lundbeck Foundation and the Novo Nordisk Foundation and administered by the Danish Regions, (grant number: R155-2014-2647) and also supported by an unrestricted grant from the Lundbeck Foundation (MEPRICA) (grant number: R155-2012-11280). JC was supported by the Danish Epilepsy Association and the Novo Nordisk Foundation (grant number NNF160C0019126).

Competing interests $\mathrm{JC}$ reported receiving honoraria for serving on the scientific advisory boards of UCB Nordic and Eisai $A B$; receiving lecture honoraria from UCB Nordic and Eisai $A B$; investigator for clinical studies for Eisai $A B$, UCB Nordic and Novartis, Pfizer and receiving travel funding from UCB Nordic.

Ethics approval The study was approved by the Danish Data Protection Agency (file no 2013-41-1719). According to the Committee on Health Research Ethics in the Central Denmark Region, no ethical approval was needed for this study.

Provenance and peer review Not commissioned; externally peer reviewed.

Data sharing statement No additional data are available.

Open Access This is an Open Access article distributed in accordance with the Creative Commons Attribution Non Commercial (CC BY-NC 4.0) license, which permits others to distribute, remix, adapt, build upon this work non-commercially, and license their derivative works on different terms, provided the original work is properly cited and the use is non-commercial. See: http://creativecommons.org/ licenses/by-nc/4.0/

(c) Article author(s) (or their employer(s) unless otherwise stated in the text of the article) 2017. All rights reserved. No commercial use is permitted unless otherwise expressly granted.

\section{REFERENCES}

1. Centers for Disease Control and Prevention (CDC). Rates of hospitalization related to traumatic brain injury-nine states, 2003. MMWR Morb Mortal Wkly Rep 2007;56:167-70.

2. Thornhill S, et al. Disability in young people and adults one year after head injury: prospective cohort study. BMJ 2000;320:1631-5.

3. Eskesen V, Springborg JB, Unden J, et al. [Guidelines for the initial management of adult patients with minimal to moderate head injury]. Ugeskr Laeger 2014;176:176.

4. Undén J, Ingebrigtsen T, Romner B, et al. Scandinavian guidelines for initial management of minimal, mild and moderate head injuries in adults: an evidence and consensus-based update. BMC Med 2013;11:50.

5. Carroll LJ, Cassidy JD, Cancelliere C, et al. Systematic review of the prognosis after mild traumatic brain injury in adults: cognitive, psychiatric, and mortality outcomes: results of the International Collaboration on Mild Traumatic Brain Injury Prognosis. Arch Phys Med Rehabil 2014;95:S152-S173.
6. Cushman JG, Agarwal N, Fabian TC, et al. Practice management guidelines for the management of mild traumatic brain injury: the EAST practice management guidelines work group. J Trauma 2001;51:1016-26.

7. Barbosa RR, Jawa R, Watters JM, et al. Evaluation and management of mild traumatic brain injury: an Eastern Association for the Surgery of Trauma practice management guideline. J Trauma Acute Care Surg 2012;73:S307-14.

8. Kraus JF, Hsu P, Schafer K, et al. Sustained outcomes following mild traumatic brain injury: results of a five-emergency department longitudinal study. Brain Inj 2014;28:1248-56.

9. Binder LM, Rohling ML, Larrabee GJ. A review of mild head trauma. Part I: Meta-analytic review of neuropsychological studies. J Clin Exp Neuropsychol 1997;19:421-31.

10. Binder LM. A review of mild head trauma. Part II: Clinical implications. J Clin Exp Neuropsychol 1997;19:432-57.

11. Rohling ML, Binder LM, Demakis GJ, et al. A meta-analysis of neuropsychological outcome after mild traumatic brain injury: re-analyses and reconsiderations of Binder et al. (1997), Frencham et al. (2005), and Pertab et al. (2009). Clin Neuropsychol 2011;25:608-23.

12. Frencham KA, Fox AM, Maybery MT. Neuropsychological studies of mild traumatic brain injury: a meta-analytic review of research since 1995. J Clin Exp Neuropsychol 2005;27:334-51.

13. Kraus J, Hsu P, Schaffer K, et al. Preinjury factors and 3-month outcomes following emergency department diagnosis of mild traumatic brain injury. J Head Trauma Rehabil 2009;24:344-54.

14. Faux S, Sheedy J, Delaney R, et al. Emergency department prediction of post-concussive syndrome following mild traumatic brain injury-an international cross-validation study. Brain Inj 2011;25:14-22.

15. Innocenti F, Del Taglia B, Coppa A, et al. Quality of life after mild to moderate trauma. Injury 2015;46:902-8.

16. McMahon P, Hricik A, Yue JK, et al. Symptomatology and functional outcome in mild traumatic brain injury: results from the prospective TRACK-TBI study. J Neurotrauma 2014;31:26-33.

17. Barker-Collo S, Jones K, Theadom A, et al. Neuropsychological outcome and its correlates in the first year after adult mild traumatic brain injury: a population-based New Zealand study. Brain Inj 2015;29:1604-16.

18. Ryan LM, Warden DL. Post concussion syndrome. Int Rev Psychiatry 2003;15:310-6.

19. Bazarian JJ, Blyth B, Cimpello L. Bench to bedside: evidence for brain injury after concussion-looking beyond the computed tomography scan. Acad Emerg Med 2006;13:199-214.

20. Gosselin N, Bottari C, Chen JK, et al. Evaluating the cognitive consequences of mild traumatic brain injury and concussion by using electrophysiology. Neurosurg Focus 2012;33:e7.

21. Kristman VL, Cote P, Hogg-Johnson S, et al. The burden of work disability associated with mild traumatic brain injury in ontario compensated workers: a prospective cohort study. Open Occup Heal Saf J 2010;2:1-8.

22. Doctor JN, Castro J, Temkin NR, et al. Workers' risk of unemployment after traumatic brain injury: a normed comparison. J Int Neuropsychol Soc 2005;11:747-52.

23. Pedersen CB, Gøtzsche H, Møller JO, et al. The Danish civil registration system. A cohort of eight million persons. Dan Med Bull 2006;53:441-9.

24. Andersen TF, Madsen M, Jørgensen J, et al. The Danish National hospital register. A valuable source of data for modern health sciences. Dan Med Bull 1999;46:263-8.

25. Andersen JS, Olivarius NF, Krasnik A. The Danish National health service register. Scand J Public Health 2011;39:34-7.

26. Sundhedsdata-styrelsen. Lægemiddelstatistikregisteret (LSR). http: //sundhedsdatastyrelsen.dk/da/registre-og-services/om-de-nationa le-sundhedsregistre/sygedomme-laegemidler-og-behandlinger/ laegemiddelstatistikregisteret (accessed 21 Feb 2017).

27. Statistics Denmark. Data for research. http://www.dst.dk/en/TilSalg/ Forskningsservice\# (accessed 10 Feb 2017).

28. National Center for Health Statistics. ICD - ICD-10-CM - Internationa classification of diseases, tenth revision, clinical modification. http:// www.cdc.gov/nchs/icd/icd10cm.htm

29. Kjaersgaard MI, Vedsted P, Parner ET, et al. Algorithm linking patients and general practices in Denmark using the Danish National Health Service Register. Clin Epidemiol 2016;8:273-83.

30. ICD-10 Version:2016. International statistical classification of diseases and related health problems 10th Revision (ICD-10)-WHO Version. 2016 http://apps.who.int/classifications/icd10/browse/2016/ en\#/S06.0 (accessed 10 Feb 2017).

31. Prior A, Fenger-Grøn M, Larsen KK, et al. The association between perceived stress and mortality among people with multimorbidity: 
A prospective population-based cohort study. Am J Epidemiol 2016:184:199-210.

32. Kennedy JE, Jaffee MS, Leskin GA, et al. Posttraumatic stress disorder and posttraumatic stress disorder-like symptoms and mild traumatic brain injury. J Rehabil Res Dev 2007;44:895-920.

33. Feigin VL, Theadom A, Barker-Collo S, et al. Incidence of traumatic brain injury in New Zealand: a population-based study. Lancet Neurol 2013;12:53-64.

34. DeKosky ST, Ikonomovic MD, Gandy S, et al. Traumatic brain injury-football, warfare, and long-term effects. N Engl J Med 2010;363:1293-6.

35. Cassidy JD, Carroll LJ, Peloso PM, et al. Incidence, risk factors and prevention of mild traumatic brain injury: results of the WHO Collaborating Centre Task Force on Mild Traumatic Brain Injury. $J$ Rehabil Med 2004:28-60.

36. Ponsford J, Willmott $\mathrm{C}$, Rothwell $\mathrm{A}$, et al. Factors influencing outcome following mild traumatic brain injury in adults. $J$ Int Neuropsychol Soc 2000;6:568-79.
37. Thompson AE, Anisimowicz $\mathrm{Y}$, Miedema B, et al. The influence of gender and other patient characteristics on health care-seeking behaviour: a QUALICOPC study. BMC Fam Pract 2016;17:38.

38. Nabalamba A, Millar WJ. Going to the doctor. Health Rep 2007;18:23-35

39. Whitnall L, McMillan TM, Murray GD, et al. Disability in young people and adults after head injury: 5-7 year follow up of a prospective cohort study. J Neurol Neurosurg Psychiatry 2006;77:640-5.

40. Reuben A, Sampson P, Harris AR, et al. Postconcussion syndrome (PCS) in the emergency department: predicting and pre-empting persistent symptoms following a mild traumatic brain injury. Emerg Med J 2014:31:72-7.

41. Ruff RM. Mild traumatic brain injury and neural recovery: rethinking the debate. NeuroRehabilitation 2011;28:167-80.

42. Nguyen R, Fiest KM, McChesney J, et al. The international incidence of traumatic brain injury: a systematic review and meta-analysis. Can J Neurol Sci 2016;43:774-85. 